which reported inflammatory alterations (high levels of CSF proteins in 2 subjects and oligoclonal bands in 4 patients).

Median Erythrocyte sedimentation rate (ESR) was $12 \mathrm{~mm} / \mathrm{h}$ [IQR 18] while median C- Reactive protein was $0.34 \mathrm{mg} / \mathrm{dL}$ [IQR 0.32]. There was not complement deficiency in any patient. With regard to the tested autoantibodies, 2 subjects showed also anti-La positivity. Out of the 4 patients tested for anti-AQP4 none was positive, even if 2 patients had NMODSD.

Conclusion: Anti-SSA positive may show a wide spectrum of neurological manifestations; CNS involvement was not associated to anti-AQP4 positivity in our cohort, even in patients with NMO/NMOSD. Further investigations are required to better disclose this association and to search for novel autoantibodies.

Disclosure of Interests: None declared

DOI: 10.1136/annrheumdis-2019-eular.5588

\section{FRI0259 SEROLOGICAL IMMUNE ABNORMALITIES ASSOCIATE WITH SPECIFIC PATHOLOGICAL ACTIVE LESIONS IN LUPUS NEPHRITIS}

Takeshi Zoshima, Satoshi Hara, Mitsuhiro Kawano. Kanazawa University Hospital, Kanazawa, Japan

Background: Serological immune abnormalities such as anti-double strand DNA (dsDNA) antibodies (Abs) and hypocomplementemia $(\mathrm{HC})$ are characteristic of lupus nephritis (LN). International Society of Nephrology/Renal Pathology Society (ISN/RPS) Classification of LN defines pathological active lesions (ALs) as including endocapillary hypercellularity, karyorrhexis, fibrinoid necrosis, cellular/fibrocellular crescents, and wire-loop lesion/hyaline thrombi [Reference 1]. Few reports have focused on the clinicopathological impact of serological immune abnormalities on pathological ALs.

Objectives: To identify the clinicopathological association between serolog ical immune abnormalities and pathological ALs in LN.

Methods: We enrolled 126 Japanese LN patients who were subjected to renal biopsy in 11 hospitals from 2000 to 2018. We determined various clinical parameters at the time of renal biopsy, including creatinine (Cr), estimated glomerular filtration rate (eGFR), total protein (TP), $\lg$, $\lg A$, IgM, C3, C4, CH50, anti-nuclear antibodies (Abs), anti-double strand DNA (dsDNA) Abs, anti-Sm Abs, anti-RNP Abs in the sera, urinalysis findings, presence of comorbidities (antiphospholipid antibody syndrome, hypertension, hyperlipidemia, diabetes mellitus, and hyperuricemia), and use of any immunosuppressive medications before renal biopsy. Renal biopsy findings were classified by ISN/RPS Classification including ALs. Immune deposits were evaluated by immunofluorescence. Elevation of serum antidsDNA Abs level [dsDNA Abs (+)] was defined as $>12 \mathrm{IU} / \mathrm{mL}$. HC was defined by $\mathrm{C} 3<60 \mathrm{mg} / \mathrm{dL}$. In patients of all pathological classes, we analyzed the association between serological abnormalities and ALs by univariate and multiple regression analyses.

Results: Of 126 patients (104 females; mean age 41.8 years), dsDNA Abs $(+)$ and $\mathrm{HC}(+)$ were found in $83(65.9 \%)$ and $80(63.5 \%)$, respectively. There were no significant differences in renal function, comorbidities, immune deposits or immunosuppressive medications before renal biopsy between dsDNA Abs $(+)$ and dsDNA Abs $(-)$, or between $\mathrm{HC}(+)$ and $\mathrm{HC}(-)$ patients. In pathological study, endocapillary hypercellularity, karyorrhexis, fibrinoid necrosis, cellular or fibrocellular crescents, and wireloop lesion/hyaline thrombi were found in 81 (64.3\%), $33(26.2 \%), 19$ $(15.1 \%), 51(40.5 \%)$, and $41(32.5 \%)$, respectively. dsDNA Abs (+) had a higher frequency of endocapillary hypercellularity, karyorrhexis, fibrinoid necrosis, and wire-loop lesion/hyaline thrombi than dsDNA Abs (-). HC $(+)$ had a higher frequency of endocapillary hypercellularity, karyorrhexis, fibrinoid necrosis, cellular or fibrocellular crescents, and wire-loop lesion/ hyaline thrombi than $\mathrm{HC}(-)$. On multiple regression analysis dsDNA Abs and C3 associated with fibrinoid necrosis and karyorrhexis, respectively $(\beta=0.25, p=0.049 ; \beta=-0.31 p=0.01)$.

Conclusion: Serum anti-dsDNA Abs and $\mathrm{HC}$ were associated with fibrinoid necrosis and karyorrhexis, respectively. These results document that individual serological immune abnormalities associate with specific $A L$, suggesting that pathophysiological differences underlie each AL.

\section{REFERENCES:}

[1] J Am Soc Nephrol. 2004;15:241-50.

Disclosure of Interests: None declared

DOI: 10.1136/annrheumdis-2019-eular.4482

\section{Vasculitis}

\section{FRI0260 \\ THE INPATIENT BURDEN OF TAKAYASU'S ARTERITIS IN THE UNITED STATES: A NATIONWIDE ANALYSIS}

Catalina Sanchez Alvarez ${ }^{1}$, Paul Kroner ${ }^{2}$, Andy Abri ${ }^{3}$, Cynthia S. Crowson ${ }^{1}$ Kenneth J. Warrington'. ${ }^{1}$ Mayo Clinic, Rheumatology, Rochester, United States of America; ${ }^{2}$ Mayo clinic, Gastroenterology, Jacksonville, United States of America;

${ }^{3}$ Mayo Clinic, Rheumatology, Jacksonville, United States of America

Background: Takayasu's Arteritis (TAK) is a rare granulomatous inflammation of the large vessels. Its incidence has been reported to be 2-3 cases per million people in the United States. To our knowledge an inpatient nationwide study of TAK in the United States has not been conducted to date. The aim was to evaluate the demographic characteris tics, reason for admission, procedures, mortality and resource utilization of patients with TAK using a national database.

Objectives: The primary goal was to estimate the national inpatient prevalence of admissions involving patients with TAK. Secondary outcomes were top reasons for admission, mortality, morbidity, resource utilization, length of hospital stay (LOS), and total hospitalization charges and costs, adjusted for inflation using the Consumer-Price Index.

Methods: All patients with a principal ICD9 diagnosis code for TAK were included using the NIS 2013 and 2014, the largest publically available inpatient database in the US. Controls were 1:1 matched using a propensity score including age, gender, and Charlson Comorbidity Index (CCl). Multivariate logistic regression models were used to adjust for race, sex, patient zip code, income, $\mathrm{CCl}$, hospital region, location, size and teaching status.

Results: Of a total of 2,840 hospital admissions for patients with TAK, 2,680 were propensity-matched to admissions without TAK and included in the study. Mean age was 49 years, $81 \%$ were female. The inpatient prevalence of TAK was $4.6 / 100,000$ admissions. After adjusting for confounders, patients with TAK had propensity-matched mortality odds of $1.44(p=0.43)$ when compared to patients without TAK. The top reasons for admission were large vessel vasculitis, sepsis secondary to pneumonia and urinary tract infection, carotid artery stenosis/occlusion, and arthralgias. For secondary outcomes, patients with TAK displayed increased odds of stroke, aortic aneurysm, peripheral vascular disease and aortic valvulopathy when compared to patients without TAK. When $\mathrm{CCl}$ was evaluated it was noted that all the patients had an index lower than 4 and the great majority lower than 2 which could be related to the young age of this population. Despite the low $\mathrm{CCl}$, these patients had 7.35 days of in-hospital stay which represented 2.1 days more when compared to non-TAK patients. For resource utilization, patients with TAK displayed higher odds of peripheral vascular intervention and arteriography when compared to patients without TAK. Patients with TAK displayed significantly higher adjusted total hospital costs, charges and LOS compared to patients without TAK

Conclusion: The inpatient prevalence was higher than suspected and could be related to the severity of the disease and complications associated with TAK. TAK was associated with an increased risk of stroke, aortic aneurysm, peripheral vascular disease and vascular interventions, but no increase mortality in this group of patients. The costs associated with hospitalization were substantial.

\section{REFERENCES:}

[1] Watanabe Y, Miyata T, Tanemoto K. Current Clinical Features of New Patients With Takayasu Arteritis Observed From Cross-Country Research in Japan: Age and Sex Specificity. Circulation. 2015;132(18):1701-9.

[2] Schmidt J, Kermani TA, Bacani AK, Crowson CS, Cooper LT, Matteson EL, et al. Diagnostic features, treatment, and outcomes of Takayasu arteritis in a US cohort of 126 patients. Mayo Clinic proceedings. 2013;88 (8):822-30.

Disclosure of Interests: None declared DOI: 10.1136/annrheumdis-2019-eular.117 